\title{
Comunicación
}

\section{PARÁSITOS HELMINTOS EN Boa constrictor, Epicrates cenchria y Corallus caninus (Ophidia: Boidae) CRIADAS EN CAUTIVERIO}

\author{
Nofre Sánchez P. ${ }^{1,2}$, Manuel Tantaleán V. ${ }^{3}$, Ryan Richards G. ${ }^{4}$ y Hugo Gálvez C. ${ }^{1}$
}

\section{Abstract}

Fecal samples for the identification of helminthes were collected from three species of snakes (Boa contrictor "boa" or "mantona", Epicrates cenchria "mantona roja" or "arco iris", Corallus caninus "boa verde" or "boa esmeralda") reared in captivity. The identified parasites, either through eggs or larva were: Kalicephalus sp., Ophiotaenia sp., Rhabdias sp., Ophidascaris sp., and Hymenolepis diminuta. The results indicated that $79 \%$ of adult female Boa constrictor and $46 \%$ of their offspring, $71 \%$ of E. cenchria and $70 \%$ of $C$. caninus were positive, either to single or multiple infections. These data showed that boas reared in captivity were highly parasitized with intestinal and pulmonary helminthes.

Key words : boa, helminthes, parasite, snake, Amazonia

Los boideos pertenecen a la familia Boidae, cuyos representantes más conocidos son las boas y los pitones. Estos reptiles no son venenosos, pero sí peligrosos por la cantidad de dientes que poseen y por su hábito constrictor. Las especies Boa constrictor ("Boa" o "Mantona"), Epicrates cenchria ("Mantona roja"o "Arco iris") y Corallus caninus ("Boa verde" o "Boa esmeralda") se crían en cautiverio con fines comerciales, orientadas a un mercado para mascotas. La zoocría de boideos es una actividad poco desarrollada en el país por lo que se conoce poco acerca de su estado sanitario; aunque los parásitos, principalmente intestinales, son reportados como casuística de mortalidad y morbilidad.
En el Perú, son escasas las referencias sobre fauna helmintológica de serpientes. Tantaleán (1976), Tantaleán y Gozalo (1985, 1992) y Severino et al. (1996) reportan diversos parásitos en Xenodon sp., Bothrops atrox, Crotalus durissus y Lachesis muta mantenidos en cautiverio. Todos estos trabajos se han realizado en ofidios de las familias Viperidae y Colubridae, no habiendo estudios locales en la familia Boidae. El presente trabajo tuvo por objetivo determinar la presencia de helmintos en tres especies de boideos manejados en cautividad en un zoocriadero autorizado de la ciudad de Iquitos.

\footnotetext{
${ }^{1}$ Estación Experimental del Centro de Investigaciones IVITA-Iquitos, FMV-UNMSM

${ }^{2}$ E-mail: ivitaq@terra.com.pe

${ }^{3}$ Laboratorio de Parasitología, Facultad de Ciencias y Filosofía, Universidad Peruana Cayetano Heredia

${ }^{4}$ Captive Breeding E.I.R.L.
} 
Cuadro 1. Resultados del examen coproparasitológico en tres especies de boideos de la Amazonía peruana criados en cautiverio en la zona de Iquitos (2001)

\begin{tabular}{lcccc}
\hline Especie & $\begin{array}{c}\mathrm{N}^{\mathrm{o}} \text { de } \\
\text { muestras }\end{array}$ & $\begin{array}{c}\text { Muestras } \\
\text { positivas }(\%)\end{array}$ & $\begin{array}{c}\text { Infección } \\
\text { simple }(\%)\end{array}$ & $\begin{array}{c}\text { Infección } \\
\text { mixta }(\%)\end{array}$ \\
\hline Boa constrictor, adultos & 28 & 79 & 54 & 25 \\
Boa constrictor, crías & 13 & 46 & 38 & 8 \\
Epicrates cenchria & 28 & 71 & 32 & 39 \\
Corallus caninus & 10 & 70 & 20 & 50 \\
\hline
\end{tabular}

Entre enero y abril del 2001 se colectaron 79 muestras de heces: 28 de ejemplares adiltos y 13decrías deBoa constrictor, 28 de adultos de Epicrates cenchria y 10 de adultos de Corallus caninus, correspondientes a la totalidad de la población del zoocriadero "Captive Breeding", ubicado en la carretera Zungarococha, en Iquitos, Perú. Todos los animales adultos fueron capturados en su medio natural y acondicionados al cautiverio (1-8 años), en tanto que las crías nacieron en cautiverio y tenían tres meses de edad en promedio. La alimentación fue en base a ratones y pollos bebé. Ninguna serpiente recibió tratamiento antiparasitario antes ni durante el muestreo.

Las muestras fueron colectadas de jaulas individuales, formolizadas y procesadas en el Laboratorio de la Estación Experimental del Centro de Investigaciones IVITA-Iquitos, de la Universidad Nacional Mayor de San Marcos y en el Laboratorio de Parasitología de la Facultad de Ciencias y Filosofía de la Universidad Peruana Cayetano Heredia. Las muestras se procesaron mediante los métodos directo y de sedimentación descritos por García (1983). La lectura se hizo en un microscopio de luz a 100 y 400x. La identificación de los huevos y larvas se hizo por comparación con muestras patrones.

En el Cuadro 1 se observa que el porcentaje de infección parasitaria en las tres especies de boideos es cercana al $80 \%$. La infección mixta es común, especialmente en E. cenchria y C. caninus.

El Cuadro 2 muestra las especies de parásitos encontrados en las tres especies de boideos en estudio. Tres nemátodos (Kalicephalus sp., Rhabdias sp. y Ophidascaris sp.) y dos céstodes (Ophiotaenia sp. e Hymenolepis diminuta). Tanto los adultos como las crías de $B$. constrictor estaban infectados con las mismas especies de helmintos. Un caso muy particular fue la presencia de Ophidascaris observado en Epicrates cenchria como la serpiente hospedera. Los resultados obtenidos muestran que los boideos en cautiverio están parasitados con nemátodos intestinales y pulmonares, y con céstodos. Estos boideos tienen hábitos alimenticios semejantes (Carrillo e Icochea, 1995).

Aunque los porcentajes de infección obtenidos son altos, es importante indicar que debido a que se realizó un solo muestreo por ejemplar, el resultado obtenido podría estar subestimando los valores reales; esto debido a que los parásitos eliminan huevos periódicamente y la ausencia de huevos no evidencia ausencia de parásitos.

Kalicephalus sp. es un nemátodo común del estómago e intestino de los ofidios. Tiene ciclo directo y la infección es provocada por la ingestión de la larva 
Cuadro 2. Parásitos helmintos encontrados en tres especies de boideos de la Amazonía peruana criados en cautiverio en la zona de Iquitos (2001)

\begin{tabular}{|c|c|c|c|c|c|c|c|c|}
\hline \multirow{3}{*}{ Especie } & \multicolumn{4}{|c|}{ Boa constrictor } & \multirow{2}{*}{\multicolumn{2}{|c|}{ E. cenchria }} & \multirow{2}{*}{\multicolumn{2}{|c|}{ C. caninus }} \\
\hline & \multicolumn{2}{|c|}{ Adulto } & \multicolumn{2}{|c|}{ Cría } & & & & \\
\hline & $\mathrm{n}$ & $\%^{1}$ & $\mathrm{n}$ & $\%$ & $\mathrm{n}$ & $\%$ & $\mathrm{n}$ & $\%$ \\
\hline Ophidascaris sp. & 0 & 0 & 0 & 0 & 1 & 4 & 0 & 0 \\
\hline Kalicephalus sp. & 4 & 14 & 1 & 8 & 7 & 25 & 5 & 50 \\
\hline Rhabdias sp. & 9 & 32 & 2 & 15 & 16 & 54 & 6 & 60 \\
\hline Ophiotaenia sp. & 16 & 64 & 1 & 8 & 5 & 18 & 3 & 30 \\
\hline H. diminuta & 3 & 11 & 3 & 23 & 4 & 14 & 0 & 0 \\
\hline
\end{tabular}

${ }^{1}$ Referido a la población de animales positivos a helmintos dentro de cada especie de boideo

infectante o por perforación cutánea activa (Flynn, 1973; Soulsby, 1987). Se puede presentar obstrucción intestinal por la formación de granulomas (Fraser, 1993).

Las Rhabdias son nemátodos pulmonares que se presentan en ofidios, sapos y ranas. La hembra coloca huevos embrionados o larvas rhabditifomes en el pulmón, los cuales son deglutidos y salen al exterior con la materia fecal (Flynn, 1973). Las infecciones son mayormente de tipo subclínica, pero pueden estar asociadas a una neumonía bacteriana secundaria y los casos graves pueden causar la muerte (Fraser, 1993). El hallazgo de Rhabdias en las crías sugiere una infección adquirida en cautiverio. Se postula que la infección de los reptiles se realizaría por vía oral, debido a que la piel del reptil es dura y seca (Baker, 1979); lo que indicaría que la infección parasitaria de las crías se realizaría por la ingestión del alimento contaminado con las heces de los boideos adultos.

El Ophidascaris sp. agrupa a varias especies morfológicamente semejantes a los ascáridos de los mamíferos, e incluso sus huevos presentan membrana externa mamelonada, aunque su ciclo biológico es indirecto. Las serpientes los adquieren al ingerir huéspedes intermediarios como batracios o mamíferos pequeños (Flynn, 1973). Fraser (1993) indica que estos nemátodos pueden provocar lesiones severas y hasta la muerte debido a que forman conglomerados en el estómago e intestino, obstruyéndolo o perforando la pared intestinal.

Las tenias se observan en todas las órdenes de reptiles, los cuales pueden servir como huésped definitivo, paraténico o intermediario de un gran número de especies. Si bien la mayoría de ellos no son patógenos, en algunos casos se ha comunicado pérdida de peso y muerte (Fraser, 1993). La especie involucrada varía de acuerdo al huésped y a la región geográfica. La infección del huésped definitivo se produce por la ingestión de los huéspedes intermediarios que llevan la forma infectante (rana o pescado) (Flynn, 1973). Los céstodos viven muchos años sin causar algún daño visible en el hospedero, y la infección por el género Ophiotaenia es común en ofidios (Hackbarth, 1990). El hallazgo de céstodos en crías de $B$. constrictor evidencia que la teniasis en esta especie es temprana y que podría ser la causa de morbilidad y mortalidad en animales jóvenes y adultos cautivos.

Es probable que la presencia de huevos de Hymenolepis diminuta en las heces no 
tenga significación patológica, debido a que éste es un céstodo que parasita a ratas, ratones y otros roedores silvestres (Flynn,1973). El hallazgo de los huevos en la materia fecal de los boideos significa que estos pequeños mamíferos son ingeridos por el reptil, ya sea en su medio natural o en cautiverio. Es una infección esporádica y de poca importancia clínica en el hombre (Reyes y Noemí, 1991; Beck y Davies, 1983).

Estos resultados sugieren que los boideos adultos en cautiverio mantienen la fauna helmintológica adquirida en el medio natural a través de la ingestión de sus presas o por ingestión de huevos y larvas infectivas.

\section{Literatura Citada}

1. Baker, M.R. 1979. The free-living and parasitic development of Rhabdias spp. (Nematoda: Rhabdiasidae) in amphibians. Can. J. Zool. 57: 161-178.

2. Beck, J.W.; J.E. Davies. 1983. Parasitología médica. $3^{a}$ ed. p 195. Ed. Interamericana. México.

3. Carrillo, N.; J. Icochea. 1995. Lista taxonómica preliminar de los reptiles vivientes del Perú. Publ. Mus. Hist. Nat. UNMSM (A) 49: 1-27.
4. Flynn, R.J. 1973. Parasites of laboratory animal. p 159-161. Iowa State University Press. Ames, USA.

5. Fraser, C.M. 1993. El manual Merk de veterinaria. $4^{a}$ ed. p 1243. Ed. Océano. España.

6. García, Sh.L. 1983. Diagnóstico de laboratorio de las infecciones parasitarias. En: Diagnóstico microbiológico. $6^{\mathrm{a}}$ ed. $\mathrm{p}$ 446-453. Sydney, F.; M. William (eds). Ed. Médica Panamericana. Argentina.

7. Hackbarth, R. 1990. Reptile diseases. p 74-80. TFH Publications I. USA.

8. Reyes, H.; I. Noemi. 1991. Hymenolepiasis y dipilidiasis. En: Parasitología clínica. $3^{a}$ ed. A. Atías (ed). p 216-217. Ed. Mediterráneo. Chile.

9. Severino, R.; R. Martínez; F. Lazo; V. Osorio. 1996. Reporte sobre parásitos de ofidios de la Amazonía del Perú. Res. V Reunión Cient. Inst. Inv. Cienc. Biol. “Antonio Raimondi” (ICBAR). Lima. p 110.

10. Soulsby, E.J.L. 1987. Parasitología y enfermedades parasitarias en los animales domésticos $7^{\mathrm{a}}$ ed. Oficina Sanitaria Panamericana. México.

11. Tantaléan, M. 1976. Contribución al conocimiento de los helmintos de vertebrados del Perú. Biota 10: 437-443.

12. Tantaléan, M.; A. Gozalo. 1985. Parásitos de Bothrops atrox (Viperidae) de la Amazonía peruana. AMVEAP 20: 11-12 